\title{
How good are citizen weather stations? Addressing a biased opinion.
}

\author{
Simon Bell, Dan Cornford, Lucy Bastin
}

Aston University, Computer Science.

\begin{abstract}
The UK is home to a dense network of Citizen Weather Stations (CWS) primarily set up by members of the public. The majority of these stations record air temperature, relative humidity and precipitation, amongst other variables, at sub-hourly intervals. This high resolution network could have benefits in many applications, but only if the data quality is well characterised. Here we present results from an intercomparison field study, in which popular CWS models were tested against Met Office standard equipment. The study identifies some common instrumental biases and their dependencies, which will help us to quantify and correct such biases from the CWS network.
\end{abstract}

\section{Keywords}

amateur, quality, bias, uncertainty, hobbyist, user-contributed, volunteered.

\section{Introduction}

Currently over 1700 citizen weather stations (CWS) are observing and recording the weather across the UK. For comparison, the Met Office runs 250 or so land surface stations in its professional Meteorological Monitoring System, abbreviated to 'MMS' (Green, 2010). Tapping into this dense network of citizen observations could have benefits in many applications.

A citizen weather station (CWS) is defined as a weather station set up by a member of the public for whom the terms weather enthusiast, volunteer, hobbyist and amateur observer are fitting descriptions. Crucially, these stations are set up out of personal interest (or, in schools, for educational purposes) rather than because it is the owner's job. Increasingly the weather stations being used are consumer automatic weather stations which are low cost and easy to install. Once connected to an internet enabled computer they can automatically submit observations to websites such the Met Office's WOW website (wow.metoffice.gov.uk), Weather Underground (www.wunderground.com) or the Citizen Weather Observer Program (CWOP - wxqa.com) for sharing. Most users submit their observations at sub-hourly intervals, most commonly every 5 minutes. The majority of CWS are located in the owner's garden with most stations heavily clustered around urban and suburban areas.

The high temporal and spatial resolution of the freely available CWS data potentially lends itself to many applications. The data could be fed into the data assimilation scheme of a high resolution weather prediction model, used to post-process model output, analysed for urban heat island studies (Steeneveld et al. 2011, Wolters and Brandsma, 2012) or even used to estimate snow depths (Muller, 2013). 
Using CWS data in the aforementioned applications raises concerns about data quality. Can CWS really compete with accurate equipment installed by professional organisations at well calibrated and exposed sites? Uncertainty about CWS data can arise from any of the following 5 sources:

1. Calibration issues - A CWS sensor may not be perfectly calibrated. Perhaps it was biased before installation, or it has drifted over time.

2. Design flaws - Often the design of CWS makes it susceptible to inaccurate readings, particularly during certain weather conditions.

3. Communication and software errors - Can produce gross errors as well as missing data.

4. Metadata issues - Incomplete or inaccurate metadata makes data interpretation difficult.

5. Representativity error - It's difficult to assess whether CWS observations represent a scale suitable for the application.

Here we present results from an intercomparison field study, where common models of CWS are collocated alongside professional equipment, allowing us to begin quantifying the magnitude of the bias and gross errors resulting from the first two sources, which we call instrumental errors. We also discuss how this field study has helped us to model the CWS bias. This is vital for quantifying such biases, and for structuring the framework of a quality control system which enables us to reduce biases and characterise residual uncertainty, allowing principled use of CWS data in scientific applications.

\section{The Field Study}

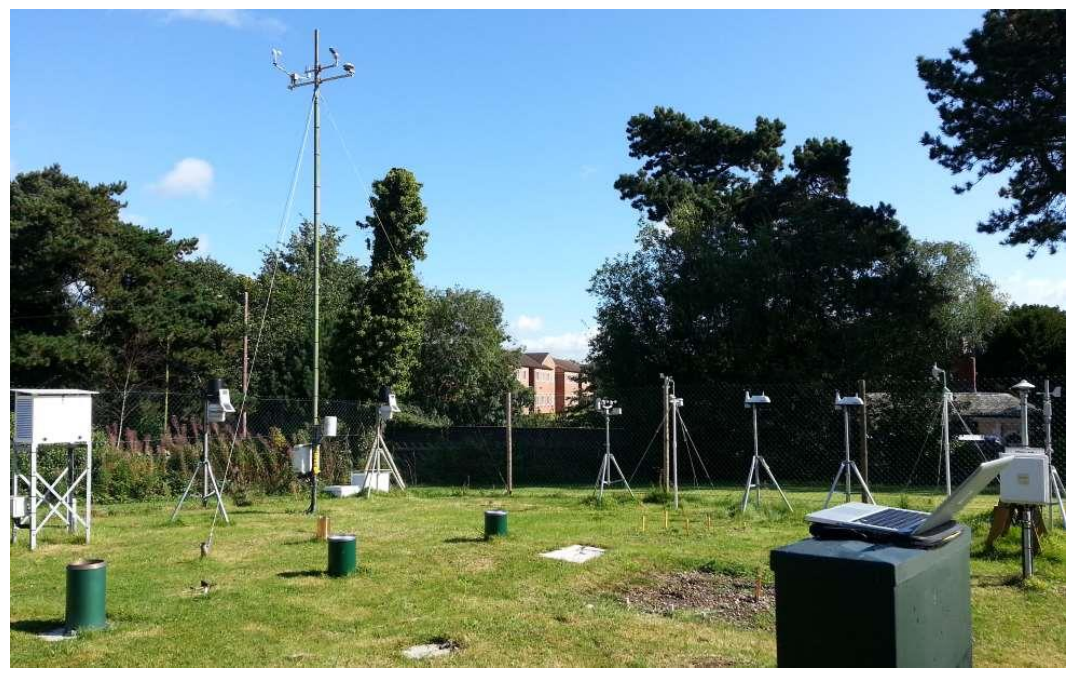

Figure 1. The Met Office's Winterbourne No. 2 weather station. The site includes sensors operated by the Met Office, the University of Birmingham, and the 7 CWS being tested as part of this study.

A year-long intercomparison field study was performed using 7 CWS collocated alongside professional Met Office equipment at the University of Birmingham's 'Winterbourne No. 2' site (Figure 1). The site, located in Edgbaston (Birmingham, UK), is part of the MMS network submitting minute-resolution data. The Met Office instruments installed at the site include a Platinum Resistance Thermometer (PRT) and a Rotronic Hydroclip both mounted within a passively ventilated 
Stevenson Screen, a Munro R100 series $0.2 \mathrm{~mm}$ tipping bucket rain gauge and a Kipp and Zonen CMP11 pyranometer. PRTs offer greater accuracy along with a more stable calibration in comparison to thermistors (Burt 2012); all 7 CWS we tested use thermistors. Henceforth these reference Met Office instruments will be referred to simply as 'MMS'. MMS instruments are calibrated on a regular managed cycle and abide by WMO standards (WMO 2008); we assume therefore that they can be used as a well characterised reference against which the 7 CWS stations can be verified. However, although this site will act as a good reference, MMS stations are not immune to problems. For example, passively ventilated Stevenson screens suffer from increased uncertainty at low wind speeds (Harrison, 2010), while tipping bucket rain gauges, such as the Munro R100, can display biases when verified against standard manual rain gauges (Burt 2012). Fortunately, the Munro R100 we used read just $+1.1 \%$ higher than a Met Office MK II 'five-inch' manual rain gauge and $+1.6 \%$ higher than another, newer, Munro tipping-bucket gauge, both collocated at the site. With this relatively small bias and a virtually complete annual dataset we can use its readings with reasonable confidence. The site is somewhat sheltered, and therefore unsuitable for Met Office wind measurements, but fortunately the University of Birmingham maintains a set of instruments at the site, including a $7 \mathrm{~m}$ mast with an anemometer and wind vane manufactured by Vector Instruments. The site's sheltered nature is similar to that of many CWS sites. The study took place from 1 September 2012 to 31 August 2013. Having envisaged that the type and magnitude of the CWS bias would depend on synoptic conditions, which vary through the year, a full year's field study was undertaken.

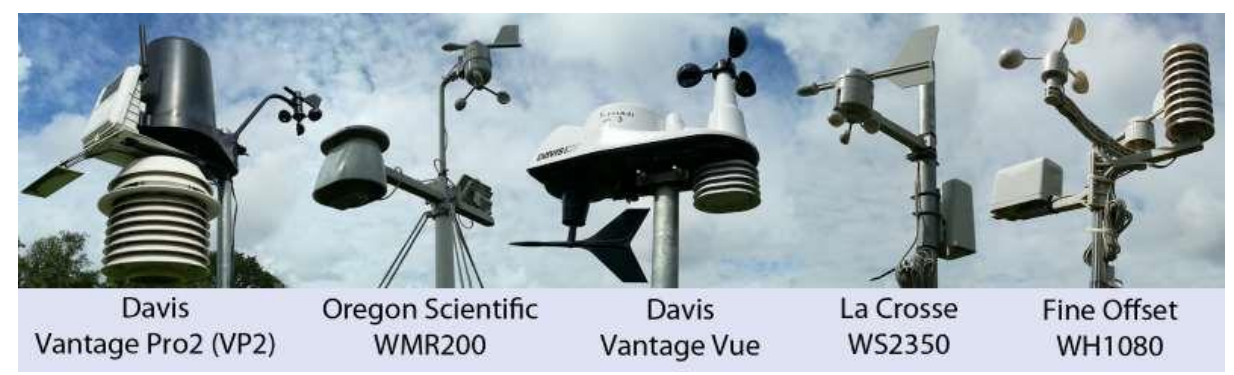

Figure 2. Citizen stations tested at the Winterbourne No.2 field site.

The 7 CWS comprised 5 different models of weather station, chosen because they are among the most popular automatic stations used by citizen observers (Bell et al., 2013). Details of the stations are summarised in Table 1, with images of the sensor suites shown in Figure 2. The two stations of each of the Davis Instruments' Vantage Pro2(VP2) and Vantage Vue, and Oregon Scientific WMR200 were installed, with the aim of identifying biases and errors common to a particular model. The second WMR200 was decommissioned in early November 2012 when its wireless transmission began to interfere with that of the first station. We are confident that there was negligible interference before this point. Only a single Fine Offset WH1080 and La Crosse WS2350 were deployed because of fears of similar interference. With hindsight, the La Crosse instruments could have used wired communications and Jenkins (2014) used two Fine Offset devices simultaneously without issue. Like most CWS, every station comprised an outdoor sensor suite and an indoor electronic console to display and store the data. Observations were downloaded from the console to a laptop on a weekly basis. All CWS and MMS temperature and humidity sensors were mounted approximately $1.5 \mathrm{~m}$ above grass. Whilst the rims of the MMS rain gauges were roughly $30 \mathrm{~cm}$ above 
grass, the height of the CWS gauges were set as recommended in their manuals, ranging between $1 \mathrm{~m}$ for the WS2350 and WMR200 to $1.5-2 \mathrm{~m}$ for the other CWS.
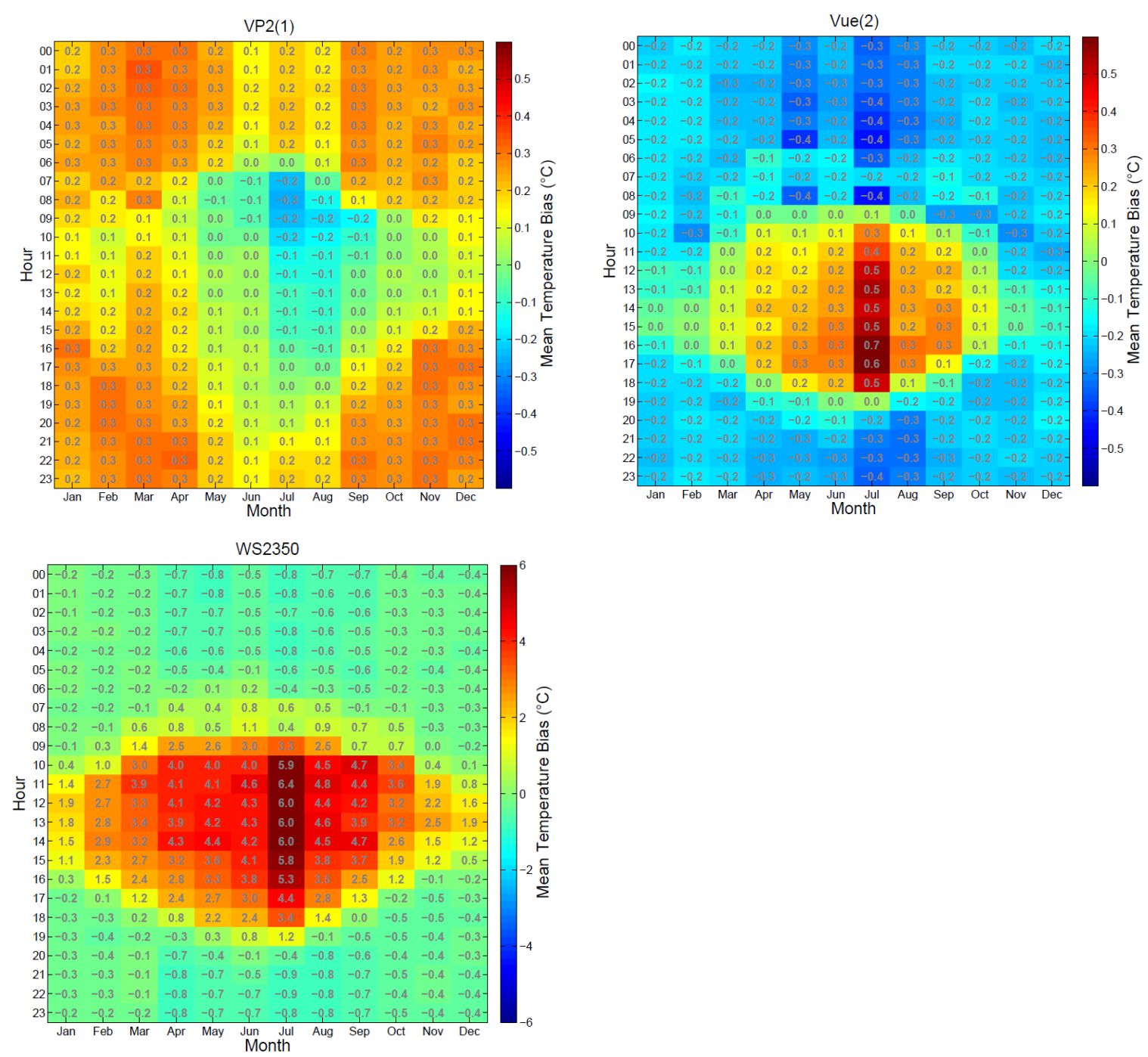

Figure 3. Mean temperature bias at different hours of the day (UTC) and months of the year for 3 of the CWS tested. a) Davis VP2(1), b) Davis Vue(2), c) La Crosse WS2350. Note the change in the colour scale for the final plot. The values written in grey are the mean bias of each cell. 


\begin{tabular}{|c|c|c|c|c|c|c|c|}
\hline $\begin{array}{l}\text { Station } \\
\text { Nickname }\end{array}$ & $\begin{array}{l}\text { Station } \\
\text { Manufacturer }\end{array}$ & $\begin{array}{l}\text { Station } \\
\text { Model }\end{array}$ & $\begin{array}{l}\text { Price }^{*} \\
\text { (Approximate) }\end{array}$ & $\begin{array}{l}\text { Software used to } \\
\text { download observations }\end{array}$ & $\begin{array}{l}\text { Temporal } \\
\text { resolution } \\
\text { (minutes) }\end{array}$ & $\begin{array}{l}\text { Time until memory full at } \\
\text { this temporal resolution } \\
\text { (days) }\end{array}$ & $\begin{array}{l}\text { Rainfall } \\
\text { increment } \\
(\mathrm{mm})\end{array}$ \\
\hline VP2(1) & $\begin{array}{l}\text { Davis } \\
\text { Instruments }\end{array}$ & $\begin{array}{l}\text { Vantage Pro2 } \\
\text { FARS }^{* *}\end{array}$ & $£ 890$ & WeatherLink & 10 & 18 & 0.2 \\
\hline $\mathrm{VP} 2(2)^{\dagger}$ & $\begin{array}{l}\text { Davis } \\
\text { Instruments }\end{array}$ & $\begin{array}{l}\text { Vantage Pro2 } \\
\text { FARS }\end{array}$ & $f 890$ & WeatherLink & 10 & 18 & 0.2 \\
\hline Vue(1) & $\begin{array}{l}\text { Davis } \\
\text { Instruments }\end{array}$ & Vantage Vue & $£ 390$ & WeatherLink & 10 & 18 & 0.2 \\
\hline Vue(2) & $\begin{array}{l}\text { Davis } \\
\text { Instruments }\end{array}$ & Vantage Vue & $£ 390$ & WeatherLink & 10 & 18 & 0.2 \\
\hline WMR200 & $\begin{array}{l}\text { Oregon } \\
\text { Scientific }\end{array}$ & WMR200 & $£ 350$ & Virtual Weather Station & 10 & 291 & 1.016 \\
\hline WS2350 & La Crosse & WS2350 & $£ 100$ & Heavy Weather & 60 & 7 & 0.518 \\
\hline WH1080 & Fine Offset $^{+\dagger}$ & WH1080 & $£ 70$ & EasyWeather & 10 & 30 & 0.3 \\
\hline
\end{tabular}

Table 1. Summary of the 7 CWS tested as part of this field study.

* Prices include accompanying software, but not mounting accessories such as tripods. Only the WMR200 comes with a mounting pole as standard. Prices include VAT.

** FARS stands for Fan Aspirated Radiation Shield.

${ }^{\dagger}$ The VP2(2) had been in the field for approx. 1 year before installation at Winterbourne No. 2. All other stations were brand new.

${ }^{+\dagger}$ Fine Offset manufacturer this station but it is frequently sold under many different brand names including Maplin, Watson, and Ambient Weather. 


\begin{tabular}{|c|c|c|c|c|c|c|c|c|c|}
\hline & \multicolumn{3}{|c|}{ Air Temperature $\left({ }^{\circ} \mathrm{C}\right)$} & \multicolumn{3}{|c|}{ Relative Humidity (\%) } & \multirow{2}{*}{$\frac{\text { Dew Point }\left({ }^{\circ} \mathrm{C}\right)}{\text { Mean Bias }}$} & \multirow{2}{*}{$\frac{\text { MSLP }^{*}(\mathrm{hPa})}{\text { Mean Bias }}$} & \multirow{2}{*}{$\begin{array}{l}\text { Rainfall } \\
\text { Absolute and percentage } \\
\text { difference from the MMS } \\
\text { yearly total of } 842.4 \mathrm{~mm}\end{array}$} \\
\hline Statistic & $\begin{array}{l}\text { Mean Bias } \\
\text { (Day and } \\
\text { Night) }\end{array}$ & $\begin{array}{l}\text { Mean Bias } \\
\text { (Day } \\
\left.\text { time }^{* *}\right)\end{array}$ & $\begin{array}{c}\text { Mean Bias } \\
\text { (Night time) }\end{array}$ & $\begin{array}{c}\text { Mean Bias } \\
\text { (all conditions) }\end{array}$ & $\begin{array}{c}\text { Mean Bias } \\
\text { (Wet conditions, } \\
>90 \%)^{\dagger}\end{array}$ & $\begin{array}{l}\text { Mean Bias } \\
\text { (Dry conditions, } \\
<=90 \%)^{\dagger}\end{array}$ & & & \\
\hline VP2(1) & $+0.2(0.2)$ & $+0.1(0.2)$ & $+0.3(0.2)$ & $+2.7(2.9)$ & $-1.3(1.2)$ & $+3.6(2.3)$ & $+0.7(0.6)$ & $+1.7(0.3)$ & $-83.4 \mathrm{~mm}(-9.9 \%)$ \\
\hline VP2(2) & $+0.2(0.3)$ & $+0.1(0.3)$ & $+0.3(0.3)$ & $+0.4(3.1)$ & $-2.2(3.1)$ & $+1.0(2.7)$ & $+0.3(0.5)$ & $+1.2(0.3)$ & $+94.8 \mathrm{~mm}(+11.3 \%)$ \\
\hline Vue(1) & $+0.1(0.3)$ & $+0.2(0.3)$ & $+0.1(0.2)$ & $+2.7(2.1)$ & $-0.2(0.9)$ & +3.4(1.7) & $+0.8(0.7)$ & +1.7(0.6) & $-22.6 \mathrm{~mm}(-2.7 \%)$ \\
\hline Vue(2) & $-0.1(0.3)$ & $+0.0(0.3)$ & $-0.2(0.2)$ & $+3.9(2.0)$ & $+1.1(0.9)$ & $+4.5(1.6)$ & $+0.8(0.7)$ & $+2.9(0.8)$ & $-28.6 \mathrm{~mm}(-3.4 \%)$ \\
\hline WMR200 & $+0.8(1.3)$ & +1.5 (1.4) & $+0.1(0.4)$ & $-\mathbf{- 1 1 . 0}(6.3)$ & $-2.8(4.1)$ & $-12.8(5.2)$ & $-1.7(1.4)$ & $+2.6(1.5)$ & $-43.8 \mathrm{~mm}(-5.2 \%)$ \\
\hline WS2350 & $+0.9(2.3)$ & $+2.1(2.5)$ & $-0.5(0.5)$ & $-1.4(5.2)$ & $-1.3(2.0)$ & $-1.4(5.7)$ & $+0.9(1.4)$ & +1.9(1.1) & $-100.0 \mathrm{~mm}(-11.9 \%)$ \\
\hline WH1080 & $+0.5(0.9)$ & $+0.9(1.0)$ & $+0.0(0.3)$ & $+7.5(3.2)$ & +5.1 (1.9) & $+8.0(3.1)$ & $+2.3(1.3)$ & $+0.0(0.7)$ & $-203.4 \mathrm{~mm}(-24.1 \%)$ \\
\hline
\end{tabular}

Table 2. Key statistics from the field study over the period 1 Sept 2012 through 31 August 2013, except for Relative Humidity and Dew Point whose statistics represent the period 16 May 2013

- 31 August 2013. The standard deviation of the difference is shown in brackets next to the values of mean bias.

* Winterbourne No. 2 site does not have MMS MSLP readings, instead observations from the Coleshill MMS site $16 \mathrm{~km}$ away were used. CWS pressure readings were set to match the Coleshill reading at the start of the period, except for the WMR200 for which the MSLP correction is based upon the elevation the user enters into the electronic console.

** Here the definition of daylight is when the MMS global radiation sensor reads greater than $0 \mathrm{Wm}^{-2}$, therefore night time is when the reading is less than or equal to $0 \mathrm{Wm}{ }^{-2}$.

${ }^{+}$As measured by the MMS humidity sensor. 


\section{Results}

Table 2 summarises some key statistics from the year-long field study. Below we discuss each weather variable in turn, namely: air temperature, humidity and dew point temperature, and rainfall. Because pressure variations are well captured by the MMS network, and CWS wind measurements will often be too localised for most applications, they are not examined in detail.

\section{Temperature}

When the air temperature measurements from the $7 \mathrm{CWS}$ were verified against the MMS measurements there were significant biases (Table 2), with clear diurnal and seasonal patterns (Figure 3). The pattern is dictated by the hours of daylight, with changes in the magnitude, and sometimes the sign, of the bias between day and night.

The Davis VP2 and Vue show the closest agreement with the MMS PRT, all with a relatively small mean bias and standard deviation. The two fan aspirated VP2s show very similar results, both tending to read too warm with overall average biases of $+0.16^{\circ} \mathrm{C}$ and $+0.18^{\circ} \mathrm{C}$. Both show an increase in this warm bias at night and a decrease during the day. The two Vues, however, do not agree. The Vue(1) tends to show a warm bias which is exacerbated in the afternoon, while the Vue(2) consistently shows a cool bias, around $-0.2^{\circ} \mathrm{C}$ at night, which only changes to a warm bias in between late morning and late afternoon for the warmer months of the year, as shown in Figure 3.

The temperature bias of the other stations is more significant, each showing a warm bias that dramatically increases during the day, and leads to a positively skewed distribution of bias for these stations. The pattern of the warm bias shown in Figure 3 for the La Crosse WS2350 station is typical for all these 3 stations, with a warm bias that peaks just after midday. During summer months the warm bias is even more pronounced, occurring for more hours of the day owing to the extended hours of insolation. These warm biases are well over $1^{\circ} \mathrm{C}$ and can climb over $4^{\circ} \mathrm{C}$ for the WMR200 and WS2350. To put these biases into context, the summer average daytime urban heat island measured in London rarely exceeds $1^{\circ} \mathrm{C}$ (Wilby et al., 2011). Without accurate bias correction it would be almost impossible to identify such an urban heat island effect using some CWS models. At night the performance of these 3 stations is much improved, for example the WMR200 and WH1080 both display a small mean bias (standard deviation) of $0.05(0.4)$ and $0.03(0.3)^{\circ} \mathrm{C}$ respectively.

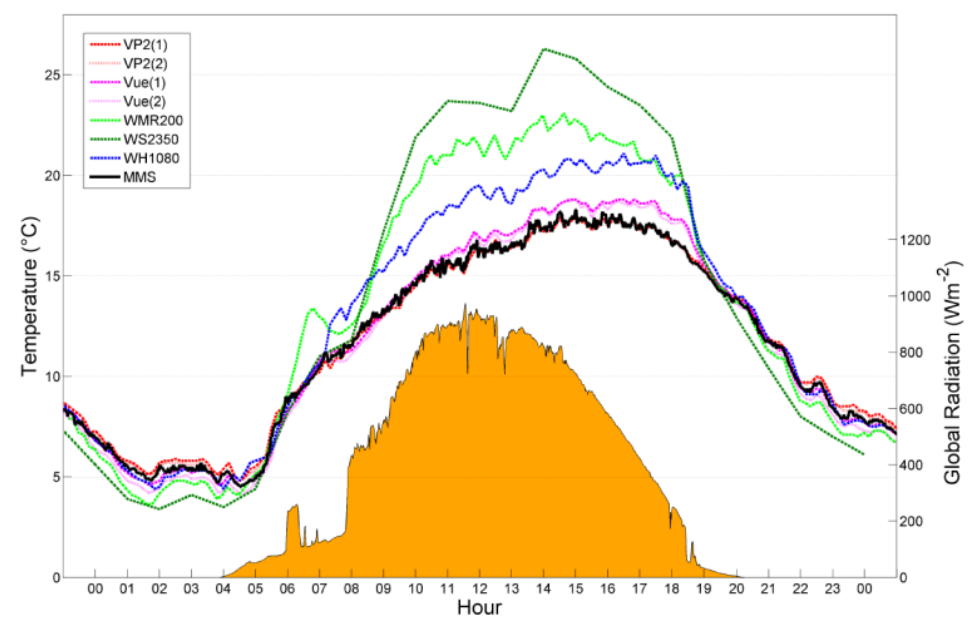

Figure 4. Time series plot of air temperature recorded by the 7 CWS and the Professional PRT housed within a Stevenson screen for 26 May 2013. A time series of MMS global radiation is shown in orange. 
Figure 4 shows a temperature time series plot for a typical day which highlights many of the patterns. For example note the large daytime warm bias exhibited by the WS2350 (and to lesser extents by the WMR200 and WH1080) and the warm bias of both Vues later in the day.

Humidity \& Dew Point

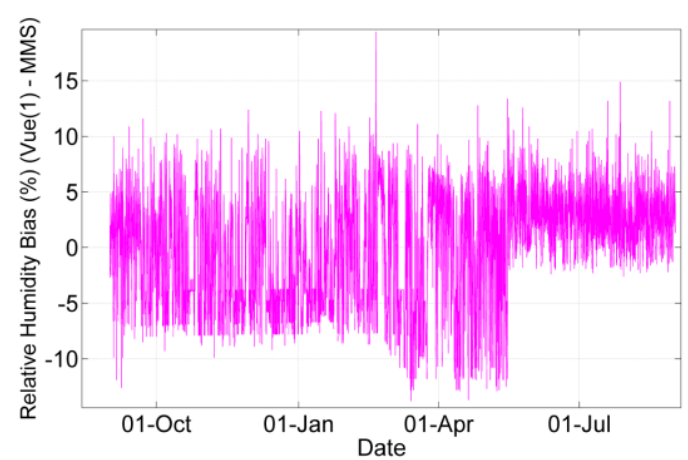

Figure 5. Time series of the Vue(1) relative humidity bias, i.e. Vue(1) humidity - MMS humidity.

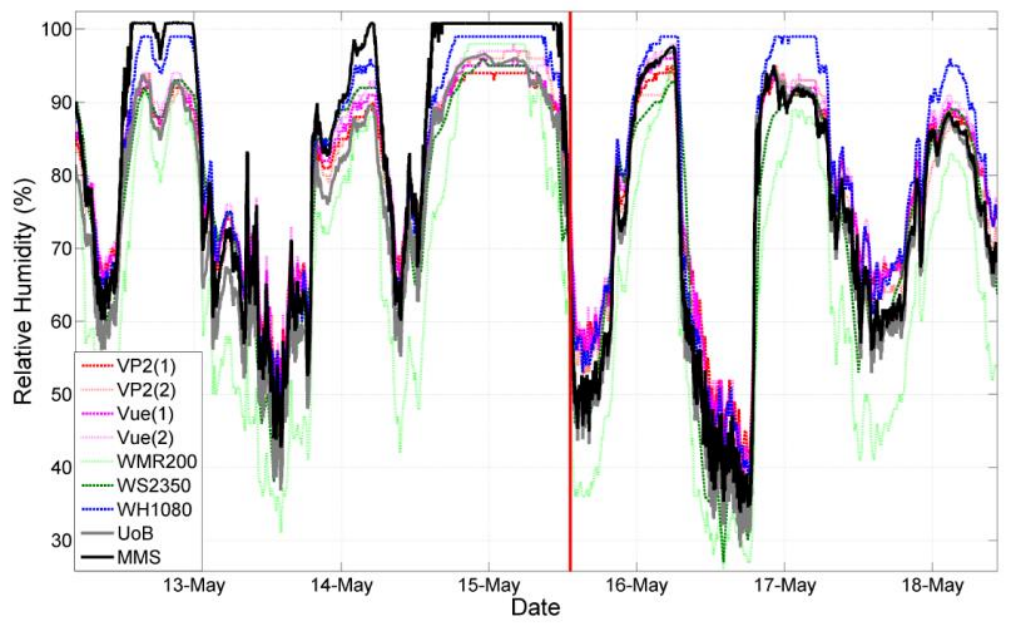

Figure 6. Relative Humidity time series. Covers the period when the MMS Hydroclip was changed, as indicated by the red line. Note the addition of the University of Birmingham (UoB) Vaisala humidity observations.

All 7 CWS tested exhibit significant relative humidity biases when compared against the MMS humidity sensor. It is important to note that there is some uncertainty associated with the MMS humidity sensor, the Rotronics Hydroclip. Figure 5 shows a time series of the Vue(1) humidity observations minus the MMS observations. The sudden step change in the bias range in mid-May is because the Hydroclip was swapped for another as part of the site's calibration process. The Hydroclip that ran over the first 8.5 months tended to read much wetter than the CWS sensors during conditions of high humidity, remaining at $100 \%$ for several hours if not days (Figure 6). The CWS observations would rarely read as high as $100 \%$. This explains the large negative biases shown in Figure 5 over this first period. The second Hydroclip showed no such tendency, exhibiting a much better agreement with a Vaisala humidity sensor run by the University of Birmingham at the site. In a separate field study, Ingleby et al. (2013) found that Hydroclip sensors tend to drift by $+1 \%$ to $+2 \%$ 
per year at Met Office sites (although there is a lot of variability) and can be slow to recover from periods stuck at saturation. They estimate that an uncertainty of $2 \%$ to $3 \%$ for an operational Rotronics Hydroclip is achievable under best conditions. As the first Hydroclip was deemed to have drifted wet, all following statistics and figures for relative humidity and dew point temperature were produced using just the second Hydroclip as the reference sensor.
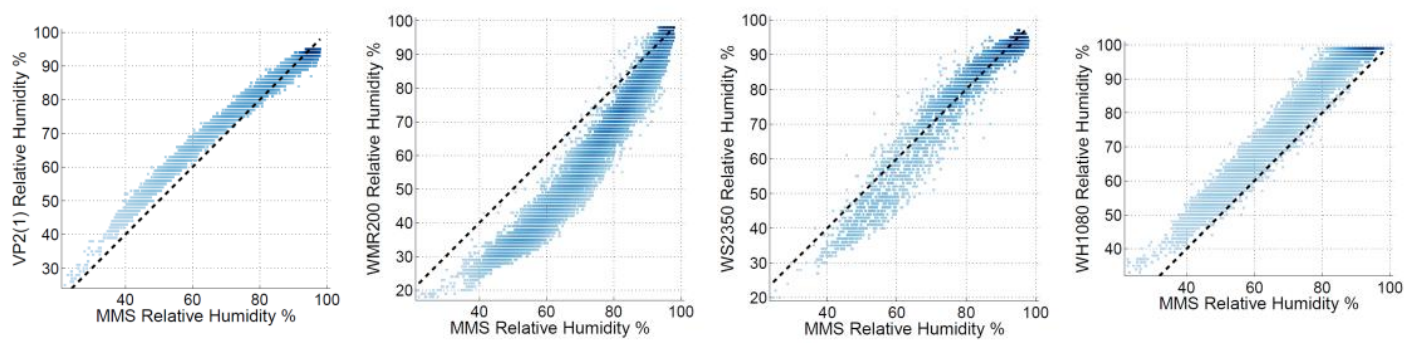

Figure 7. CWS vs. MMS relative humidity. a) VP2(1), b) WMR200, c)WS2350, d) WH1080. The darker the colour the greater the density of points.

The 7 CWS humidity sensors we tested have very different patterns to their bias. The Davis VP2(1), Vue(1) and Vue(2) all show a wet bias over the majority of the humidity range (Figure 7). For all 3 stations the mean bias is greater than $3 \%$ under drier conditions (less than $90 \%$ ), but when the humidity is greater than $90 \%$ the VP2(1) and Vue(1) exhibit small dry biases. These findings agree well with those of Burt during his review of the VP2 (Burt 2009) and Vue model (Burt 2013). The VP2(2) behaves slightly differently; under drier conditions it does not over-read to the same degree as the other Davis stations, but it under-reads more during wet conditions, with a greater residual variance across the whole humidity range.

The WMR200 under-reads across the entire humidity range, and dramatically so in drier situations where it exhibits a mean bias of $-12.8 \%$. The WS2350 also tends towards a dry bias during drier situations, but with a less extreme mean of $-1.4 \%$. Between $70-90 \%$ (Figure 7 ) this switches to a wet bias. By contrast, the $\mathrm{WH} 1080$ has a large wet bias over the entire humidity range, with an overall bias of $7.5 \%$.
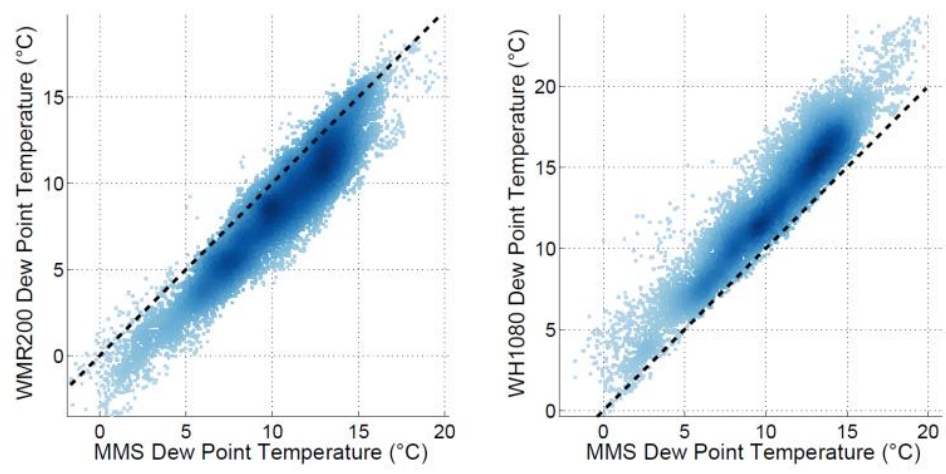

Figure 8. CWS vs. MMS dew point temperature. a) WMR200, b) WH1080. The darker the colour the greater the density of points. 
Since we anticipated some interaction of temperature and relative humidity biases, we also considered dew point temperature. The mean dew point biases for all Davis stations were within $1^{\circ} \mathrm{C}$ of the MMS. In agreement with other studies that found VP2 monthly means mostly within $1^{\circ} \mathrm{C}$ of the reference sensor (Burt, 2009) and Vue readings that were approximately $1^{\circ} \mathrm{C}$ too high (Burt, 2013), both Vues in this study had a mean bias of $+0.8^{\circ} \mathrm{C}$. The WS2350, with a mean bias of $0.9^{\circ} \mathrm{C}$ was also within $1^{\circ} \mathrm{C}$ of the MMS sensor, but with a larger residual variance. The mean bias of the WMR200 and WH1080 was more significant, at $-1.8^{\circ} \mathrm{C}$ and $2.3^{\circ} \mathrm{C}$ respectively (Figure 8 ).

The CWS dew point values were derived by the station's electronic console, however the difference due to their use of potentially different algorithms was virtually negligible, never exceeding $0.02^{\circ} \mathrm{C}$.

Rainfall

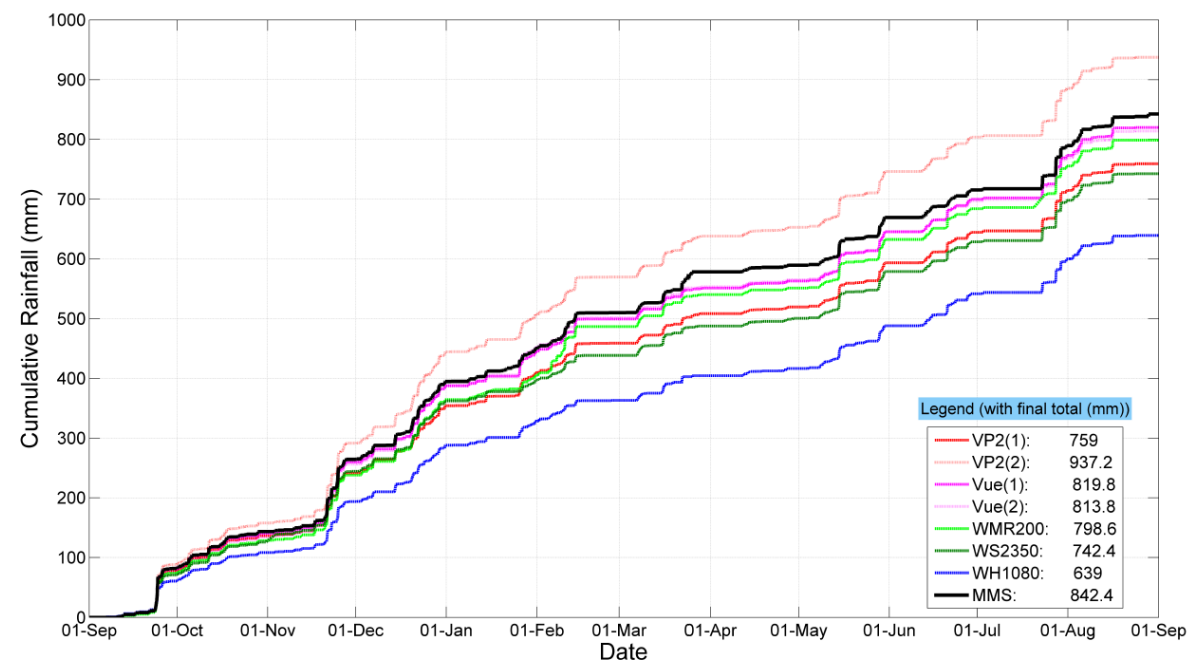

Figure 9. Cumulative rainfall totals of the $7 \mathrm{CWS}$ throughout the yearlong study period. Professional Met Office gauge is shown as the black line. The final totals are displayed in the legend.

Figure 9 shows a plot of cumulative rainfall throughout the yearlong study period. All but the VP2(2) measured totals less than the MMS gauge. It is interesting that one VP2 over-read whereas the other under-read, particularly as the VP2 model allows for calibration of the tipping buckets using a screw under each bucket. Before installing the VP2 stations they were calibrated in the lab so that on average both read within $2 \%$ of the truth. It is curious that, once in the field, they should deviate from the professional gauge by approximately $10 \%$, and in different directions. When Burt (2009) tested a different VP2 against a standard 'five-inch' gauge he found the annual total was just $1.8 \%$ higher, but the agreement was not consistent with monthly differences ranging from $-10 \%$ to $+19 \%$.

The Davis Vues show a very good agreement with the MMS gauge and with each other, both undercatching by less than $4 \%$. However, results by Burt (2013) show this slight undercatch is not consistent throughout all Vue stations; as compared to standard 'five-inch' gauge the annual total of their Vue was $9 \%$ too high. The WMR200 showed a reasonable agreement, undercatching by just $5.2 \%$ at the end of the period. The WS2350 and the WH1080 undercatch by greater amounts, with a yearly total of just $88 \%$ and $76 \%$ of the Met Office total respectively. 


\section{Parameterising station bias}

Thus far we have seen that all 7 CWS tested display some substantial biases. In this section we attempt to identify the factors determining these biases, with the aim of parameterising them.

\section{Temperature}
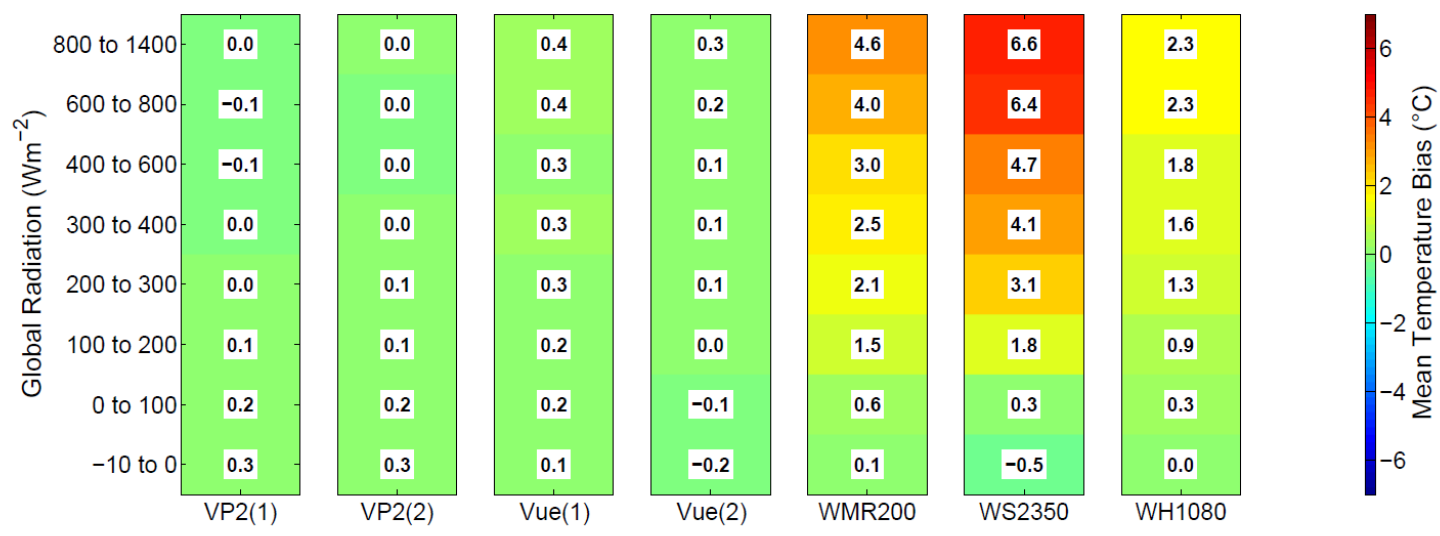

Figure 10. Temperature bias as a function of global radiation levels for the 7 CWS tested.

The most obvious pattern to the temperature bias is the difference between day and night, a result of changes in the radiative balance. For the WMR200, WS2350 and WH1080 the main driver of their daytime warm bias is the strength of incoming solar radiation. Figure 10 shows how these 3 CWS exhibit a greater warm bias with increasing levels of solar radiation. This relationship is largely linear, with some evidence of bias plateauing as global (direct + diffuse) radiation levels reach their highest levels.

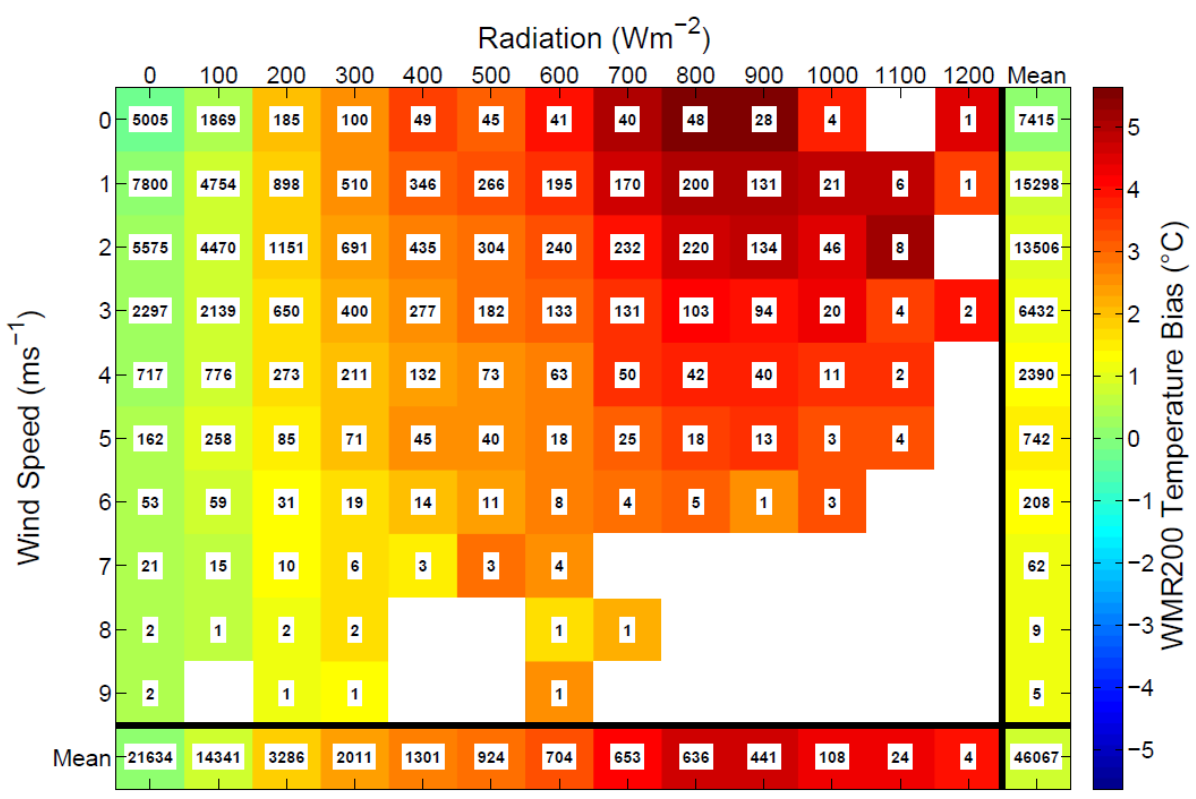

Figure 11. WMR200 temperature bias as function of wind speed and global radiation. The mean bias for a given radiation (wind speed) bin for all wind speeds(radiation levels) is shown along the bottom(right side). Here the number within each cell signifies the sample size. 
Wind speed also appears to influence this relationship. Note that in Figure 11 the warm temperature bias of the WMR200 during high solar radiation conditions is exacerbated when the wind speed is low.
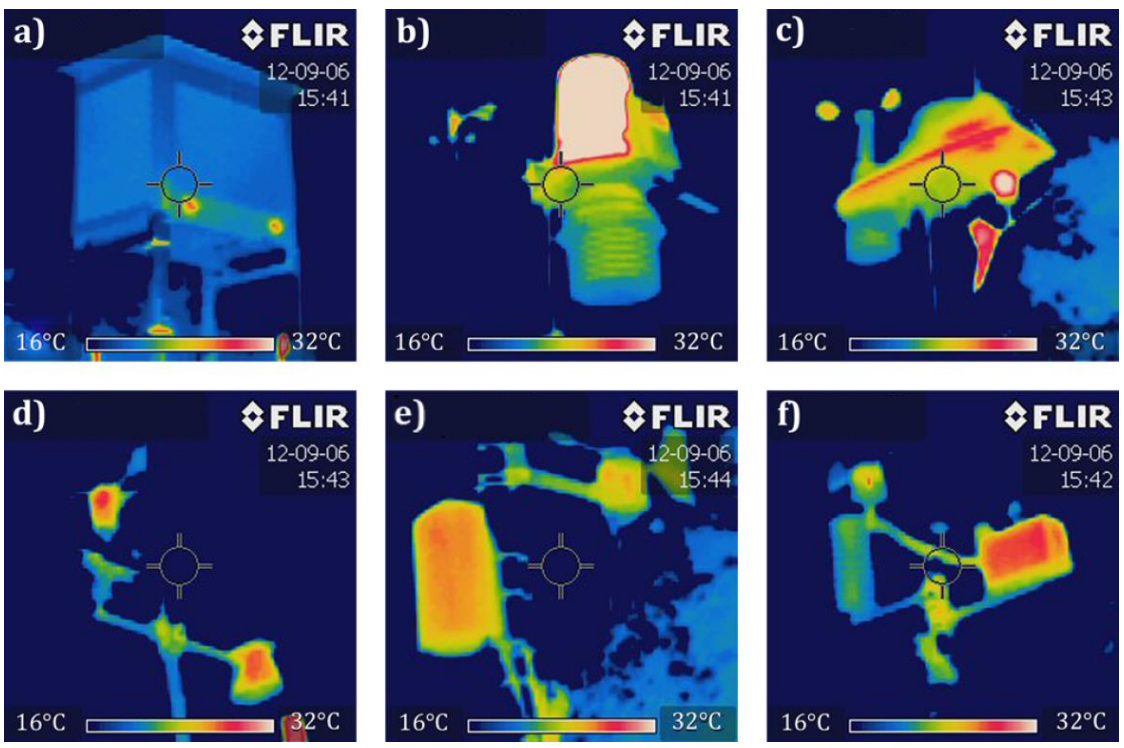

Figure 12. Thermal images taken from the South-West by a Flir i5 thermal imaging camera on a sunny summer afternoon. a) Stevenson Screen, b) VP2, c) Vue, d) WMR200, e)WS2350, f) WH1080. The colour-scale is consistent. The white (hot) part of the VP2 evident in panel $b$ is its black rain gauge. For help identifying the parts of each station cross reference with Figure 2, but be aware of the change in perspective.

All of the stations tested have some form of shielding to guard their thermistor from direct sunlight. Such shielding should also allow surrounding air to ventilate through, but it is apparent that some shields are more effective than others. Thermal imaging (Figure 12) shows that the WMR200 and WS2350, which exhibit the largest biases under increased global radiation, exhibit the warmest colours on their shields. This illustrates that their thermistor shielding is prone to overheating under sunny conditions, which heats the air inside the thermistor housing increasing the sensed temperature. This overheating is also a function of ventilation; the design of WMR200 and WS2350 shields (Figure 2) make sufficient ventilation difficult, so that the air within the shield warms, rather than being refreshed with ambient air from outside the shield. The upturned-plate design of the WH1080 allows for better ventilation and is noticeably cooler than the WMR200 and WS2350. However, the bias still displays a relationship with global radiation levels, perhaps due to its small size and off-white colour. Jenkins (2014) also identified a relationship with solar radiation for the two WH1080s in their study.

The two Davis models, the VP2 and Vue, show the coolest colours in the thermal images, and their relationship with radiation is somewhat different. The VP2s were the only model of station we tested that included a Fan Aspirated Radiation Shield (FARS).The fan is solar powered, and it is evident in Figure 3 that the VP2 has the lowest bias during the day when this fan is active. Under sunny and calm conditions the aspirated VP2s probably give a better estimate of the air temperature than the passively aspirated Stevenson screens, which are prone to increased uncertainty at low wind speeds (Harrison, 2010). During the night, when the fan is inactive, ventilation can only occur passively, leading to a warm bias. The altered shield design which incorporates active ventilation has 
compromised the effectiveness of the passive ventilation at night. It is reassuring to see the similar performance of the two VP2s, providing confidence that the parameterisation would be similar for all stations of this type.

The Davis Vues appear to have a stronger relationship with outgoing longwave radiation than shortwave radiation. As the Vue's radiation shield is mounted underneath the station's main body it is well shielded from incoming solar radiation, although its effectiveness may be compromised when the solar angle is low. It is when the land surface has warmed and outgoing radiation peaks, around mid-afternoon, that the station shows the greatest warm bias (Figure 3). Unfortunately, longwave radiation is not commonly measured at MMS stations, so a proxy variable may have to be used to parameterise Vue temperature bias. The bias in the Vue temperatures demonstrated a stronger correlation with grass temperature than with global radiation.

So far we have only looked at the correlation between simultaneous CWS temperature bias and global radiation measurements. This assumes that the impact of changes in radiation on the temperature bias is instantaneous. When a lagged impact is considered this correlation often improves. For example, for the WMR200, WS2350 and WH1080, a better correlation is found when we weight a selection of previous global radiation measurements exponentially, with radiation observations nearer in time to the temperature observation weighted more heavily. The strength of the correlation varies little between using radiation observations stretching back just 30 minutes or 120 mins - the key is to consider at least some previous observations.

\section{Humidity \& Dew Point}
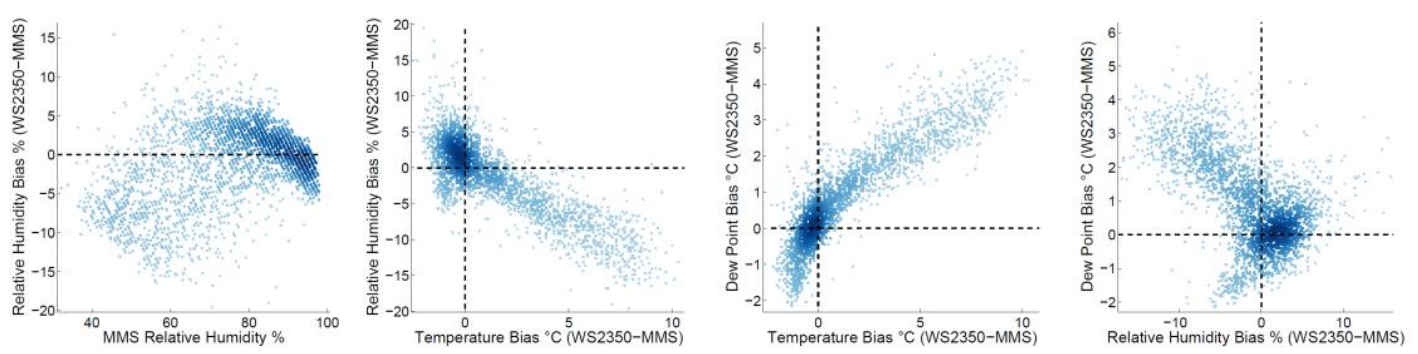

Figure 13. Plots of the relationship between temperature, humidity and dew point, and their biases for the La Crosse WS2350. The darker the colour the higher the density of points.

Parameterising biases in relative humidity is a challenging task. In general there are two main sources of bias. Firstly there is the capacitive sensor itself. Figure 7 (1:1 plots) demonstrated that in comparison to the MMS sensor CWS have potentially large calibration errors. The magnitude and even the sign of the bias will often change depending on the humidity. Bias may also be induced from hysteresis, when the sensor's response to a change in humidity varies depending on whether the humidity is rising or falling. As with the MMS Hydroclip, these CWS sensors may drift over time, potentially becoming more biased the longer they are in the field. The second source of bias comes from the inadequate shielding or housing of the sensor, and is closely related to the shielding problems that lead to temperature biases. For example if the shielding overheats, the air within the shield will also warm reducing its relative humidity, thus causing an apparent dry bias. Alternatively, if humidity is falling after a period of saturated conditions, a poorly ventilated shield may prolong the time a sensor reads saturated. Trying to tease apart the two sources of error so that they can be parameterised is very difficult. 
Figure 13 shows a series of plots for the WS2350 which can help us decipher the source of its humidity and dew point bias. It is apparent that the relationship between the humidity bias and MMS humidity is somewhat unclear. Figure 13b plots the humidity bias against temperature bias. The majority of points display a slight cold and wet bias, however there is a long tail where warm temperature biases are associated with a dry humidity bias. This may be caused by increased temperatures within the sensor housing, relative to the surrounding air. Bias in the dew point temperature will be inherited from both the humidity bias and the temperature bias. Unsurprisingly Figure 13 shows that a warm temperature bias will lead to a subsequent warm dew point bias; however, the relationship is not perfectly linear. As expected the dew point is too high when the humidity is wet biased. However, it is also too high when humidity is dry biased, perhaps because a warm temperature bias has not only caused a subsequent high dew point bias but also a dry humidity bias when the sensor housing overheated. It's worth noting that we have chosen to plot just one station here; the plots for the other stations can look very different, further indicating that the parameterisations must be learnt for each individual CWS separately.

\section{Rainfall}

6 of the 7 CWS tested displayed yearly rainfall totals lower than the MMS rain gauge. One explanation for this could have been that, at 1-2 metres above the ground, the CWS rain gauges are mounted higher than the $30 \mathrm{~cm}$ MMS gauge, as such they experience higher wind speeds which can lead to undercatch (Guo et al., 2001). In this study we looked for a relationship between wind speed and the daily CWS rainfall totals as a proportion of daily MMS total. However, no obvious relationship was found, either for average wind speed taken at times of measured rainfall, or for daily average wind speed. Measurements from both the CWS anemometer and the Vector Instruments anemometer, mounted at $7 \mathrm{~m}$, were used separately. Possible explanations for this lack of relationship is that the Winterbourne No. 2 site is relatively sheltered, with a mean $7 \mathrm{~m}$ wind speed of $1.6 \mathrm{~ms}^{-1}$ and a max of $10.2 \mathrm{~ms}^{-1}$. The anemometers of the 4 Davis instruments never read any higher than $5 \mathrm{~ms}^{-1}$. It is possible that these relatively low wind speeds did not cause noticeable undercatch and that the biases seen had a different source. The MMS gauge is deep with steep sides to prevent heavy rain from bouncing out, while all CWS tested bar the VP2s are much shallower, particularly the WS2350 and WH1080. Such a design makes them prone to undercatch due to rain drops bouncing out, an effect that potentially outweighs any influence of wind speeds.

Even before deploying the CWS rain gauges outdoors, the tipping buckets within may be poorly calibrated producing a bias straight out of the box. To test this, $500 \mathrm{ml}$ of water was slowly dripped through each rain gauge indoors. By dividing the volume of water by the area of the gauge it's possible to calculate the depth of rain in $\mathrm{mm}$ the station's console should display (Overton, 2007). Differences between expected and measured depth were as large as $13 \%$, with most stations underreading. A corresponding correction was then applied to the yearly cumulative rainfall totals. For some stations this led to a small improvement, but for others their yearly total was made much worse. Clearly other factors are at work outdoors that outweigh errors due to poor calibration, proven by the poor performance of the VP2s in the field despite being calibrated to less than $2 \%$ error indoors.

Some CWS rainfall bias, particularly when dealing with daily totals, can result from cold and snowy synoptic conditions. The gauges of the CWS tested were prone to filling with snow when the MMS 
gauge did not. This resulted in delayed tips when the snow finally melted. The funnel exit hole of the CWS gauges was also prone to freezing over, again causing a delay in rainfall readings.

\section{Using the results for modelling biases}

This experiment was not designed as a stand-alone project; the aim was to aid the construction of models that will estimate the bias and uncertainty associated with CWS observations. Here we discuss how key findings from the field study influence these models of instrumental bias.

Every CWS we tested exhibited a relationship between temperature bias and the levels of global solar radiation. The relationship varied depending on the model of station, so we'd ideally like to learn this relationship for each individual station type over time. In order to do this we would require an estimate of the solar radiation at the location of every CWS in the country.
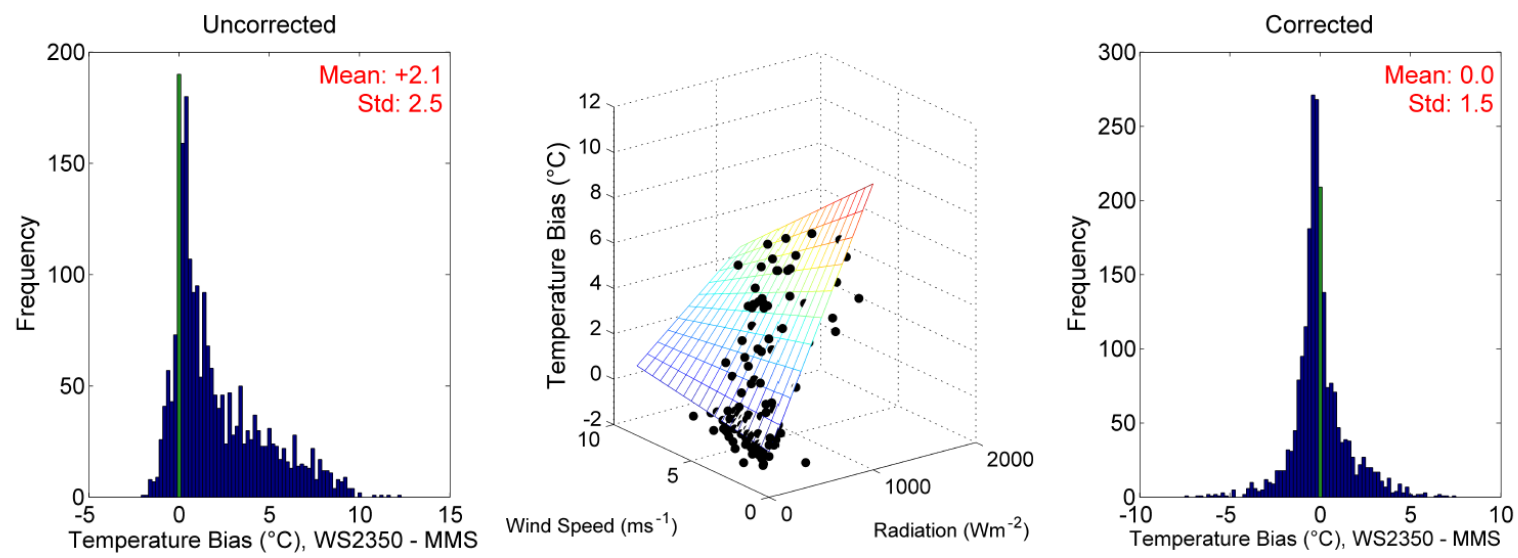

Figure 14. Demonstration of correcting CWS temperature bias using a multiple linear regression model. The figure shows a histogram of the WS2350 temperature bias before and after the correction along with a scatter plot of a sample of its observations overlaid with a grid of the learnt model. The data was randomly split in half to form the training and test datasets.

Figure 14 demonstrates that with a reliable estimate of the global solar radiation level, as is available at the test site, it is relatively simple to correct the temperature observations. Here we used a simple multiple linear regression model in which radiation, wind speed, and an interaction term were used as predictors of the temperature bias, with radiation providing most predictive power. When the temperature bias prediction was used to correct the observations there was a reduction in the mean bias and residual variance for every CWS. Sometimes this improvement was marginal, e.g. for the Davis stations, but for stations which exhibited large radiation biases, such as the WS2350, the improvement was large. As previously mentioned, the influence of radiation on temperature bias is often lagged, so incorporating previous radiation observations using an exponential weighting would improve our correction further.

We also saw that temperature bias can result from poorly calibrated sensors, as with the Vue(2) which displayed a night time cool bias of around $-0.2^{\circ} \mathrm{C}$. We can quantify this calibration bias under conditions when the bias produced by factors such as radiation and wind speed is minimal, for example at night under cloudy, breezy conditions.

The field study has reinforced that relative humidity is a difficult variable to measure, for both CWS and MMS stations. As the MMS network has its own bias that can slowly drift or suddenly jump, it is 
clear that this network may have to undergo its own quality control procedures. A network of more accurate sensors such as chilled mirror hygrometers which measure dew point temperature directly could help anchor the MMS network and in turn the CWS network, although such a network may prove difficult to keep in good operational condition. Incorporating relative humidity into our predictive model will also be difficult. With an upper limit capped at $100 \%$, it can produce nonGaussian errors. Converting relative humidity into other variables that represent air moisture, such as dew point temperature, may provide a solution to this. We have shown examples where relative humidity and dew point biases are dependent on temperature bias, so it is important that we model the moisture variable jointly with temperature and its bias.

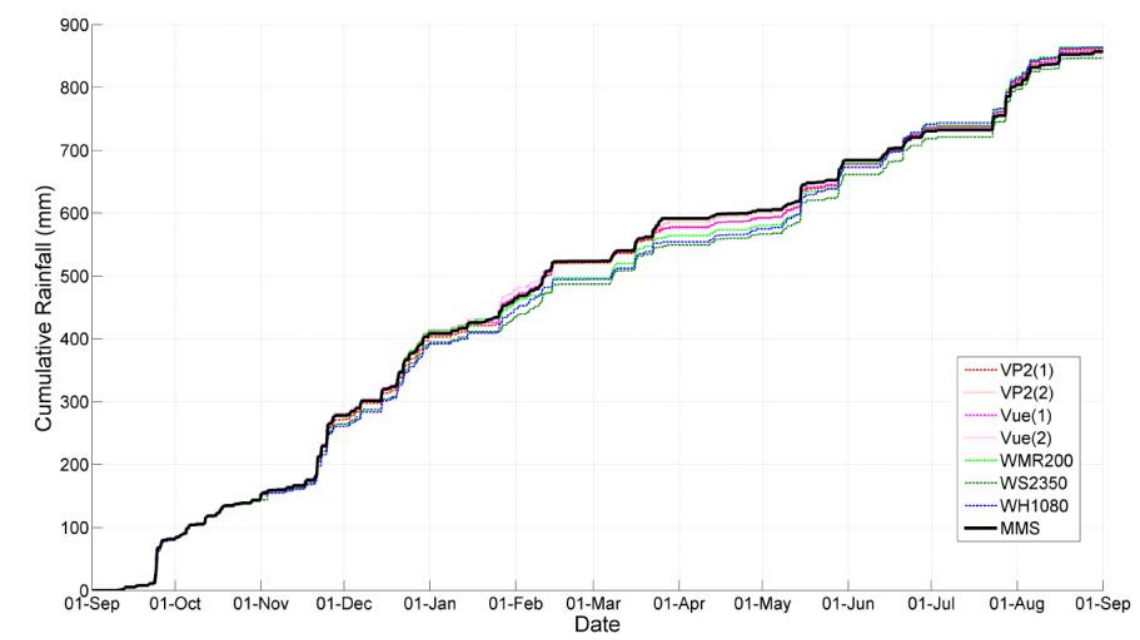

Figure 15. Plot of cumulative rainfall totals from the MMS and 7 CWS gauges. The cumulative rainfall values (available every 10 minutes) for each CWS were corrected using the relationship between their cumulative rainfall and that of the MMS gauge; learnt from preceding data only.

CWS are usually good at capturing the intensity and timing of rainfall events, but their long term cumulative total can differ from professional gauge measurements significantly. Using the MMS gauge as our best estimate of the truth, Figure 15 shows that by identifying the relationship between the MMS and CWS cumulative rainfall time series we can correct the CWS time series to fall in line with that of the MMS. This works well because, for the CWS tested here, the proportion of undercatch or overcatch tended to remain relatively constant through time; allowing for a correction to be learnt from preceding data. In reality, a MMS gauge will not be collocated alongside every CWS, in which case observations must be carefully interpolated from nearby professional gauges, e.g. from the MMS or Environmental Agency networks. Effectively merging radar accumulation data with this gauge data could improve the interpolation (DeGaetano and Wilks, 2009). This should improve CWS long term totals, whilst still keeping the detail of individual and isolated rainfall events captured by the CWS.

We've seen examples where two stations of a particular model exhibit very similar biases, for example the temperature measurements of the two VP2s, although ideally we would have tested at least 3 of each model to give higher confidence in the consistency. Station metadata becomes very valuable when it contains details such as station type, providing prior information about the bias we'd expect to see. However, as noticed by Jenkins (2014), this study also showed examples when stations of the same model actually displayed very different biases (for example the VP2s rainfall or the Vue(2)'s night time cold bias not evident in the Vue(1)). Within the full CWS network every CWS 
will have a different siting, exposure and level of calibration and maintenance. Each can introduce biases unique to a particular site. For these reasons our study is only representative of the biases in the network up to a point.

This study has shown examples of how we can correct the bias in one variable by evaluating its relationship with other variables. It is important that these relationships remain smooth through time while still being able to adapt and update. If, for example, we learn that a station has a significant warm bias under sunny conditions, we can use this to correct the temperature observations on future sunny days. However the radiation shielding may deteriorate with time, gradually exacerbating the warm radiation biases (Lopardo et al., 2013). This new relationship will need to be learnt. The presence of missing data and gross errors must also be accounted for in any future work.

\section{Conclusion}

The spatial and temporal density of data from the CWS network is appealing, and may identify weather phenomena that sparser professional networks cannot. However, as this field study has shown CWS data can contain significant instrument biases. Any application of CWS data will require a quality control system capable of not only removing gross errors but also correcting instrument bias, while also providing an uncertainty estimate. Much of the bias can be parameterised and thus learnt and corrected for, but as biases are often unique to an individual station they should primarily be learnt using the CWS data while potentially varying in time. Quantifying a station's bias will rely on obtaining a reliable estimate of the weather at CWS locations against which we can begin to verify the CWS observations and this is the subject of our future work. This will attempt to disentangle instrument biases from the natural spatial variations which we wish to capture.

\section{References}

Bell S, Cornford D, Bastin L. 2013. The state of automated amateur weather observations. Weather 68: 36-41.

Burt S. 2009. The Davis Instruments Vantage Pro2 wireless AWS - an independent evaluation against UK-standard meteorological instruments. measuringtheweather.com.

Burt S. 2012. The Weather Observer's Handbook. Cambridge University Press.

Burt S. 2013. Instrument review Davis Instruments Vantage Vue AWS. measuringtheweather.com.

DeGaetano AT, Wilks DS. 2009. Radar-guided interpolation of climatological precipitation data. International Journal of Climatology. 29: 185-196.

Green A. 2010. From Observations to Forecasts - Part 7. A new meteorological monitoring system for the United Kingdom's Met Office. Weather, 65: 272-277.

Guo JCY, Urbonas B, Stewart K. 2001. Rain Catch under Wind and Vegetal Cover Effects. Journal of Hydrologic Engineering. 6: 29-33. 
Harrison RG. 2010. Natural ventilation effects on temperatures within Stevenson screens. Quarterly Journal of the Royal Meteorological Society. 136: 253-259.

Ingleby B, Moore D, Sloan C, Dunn R. 2013. Evolution and Accuracy of Surface Humidity Reports. Journal of Atmospheric and Oceanic Technology. 30: 2025-2043.

Jenkins G. 2014. A comparison between two types of widely-used weather stations. Weather. Waiting for publication.

Lopardo G, Bertiglia F, Curci S, Roggero G, Merlone A. 2013. Comparative analysis of the influence of solar radiation screen ageing on temperature measurements by means of weather stations. International Journal of Climatology. 34: 1297-1310.

Muller CL. 2013. Mapping snow depth across the West Midlands using social media-generated data. Weather. 68. 82.

Overton AK. 2007. A guide to the siting, exposure and calibration of automatic weather stations for synoptic and climatological observations. http://myweb.tiscali.co.uk/awsguide (accessed 10 October 2013).

Steeneveld GJ, Koopmans S, Heusinkveld BG, van Hove LWA, Holtslag AAM. 2011. Quantifying urban heat island effects and human comfort for cities of variable size and urban morphology in the Netherlands. J. Geophys. Res. 116.

Wilby RL, Jones PD, Lister DH. 2011. Decadal variations in the nocturnal heat island of London. Weather. 66. 59-64.

WMO. 2008. Guide to Meteorological Instruments and Methods of Observation, Volume 8, 7th Edition. World Meteorological Organization: Geneva, Switzerland.

Wolters D, Brandsma T. 2012. Estimating the Urban Heat Island in residential areas in the Netherlands using observations by weather amateurs. J. Appl. Meteorol. Climatol. 51: 711-721.

\section{Acknowledgements}

This research is funded by an EPSRC CASE award (10002388) with the Met Office. Thanks are due to Shona Hogg and Fiona Carse at the Met Office for providing MMS data, to Mike Molyneux for supporting the field study, to Duick Young at the University of Birmingham for his help setting up the intercomparison field site and to Stephen Burt for his help interpreting the field study results. 